\title{
Structure-property relationships of polypropylene-based nanocomposites obtained by dispersing mesoporous silica into hydroxyl-functionalized polypropylene. Part 1: toughness, stiffness and transparency
}

\author{
Ryota Watanabe $\mathbb{D}^{1} \cdot$ Hideaki Hagihara $^{1} \cdot$ Hiroaki Sato ${ }^{1}$
}

Received: 13 March 2018 / Revised: 18 May 2018 / Accepted: 22 May 2018 / Published online: 26 June 2018

(c) The Author(s) 2018. This article is published with open access

\begin{abstract}
The long-range objective of this study is to provide better understanding of the relationship between the structures and properties of PP-based nanocomposites containing mesoporous silica (MPS). In this investigation, nanocomposites composed of MPSs with various porosity structures (two types of Santa Barbara Amorphous No. 15 (SBA-15) with pores of 4.4 or $8.0 \mathrm{~nm}$, and Mobile Composition of Matter No. 41 (MCM-41) with pores of $2.9 \mathrm{~nm}$ ) and polypropylene (PP) or functionalized PP containing hydroxyl groups $(\mathrm{PPOH})$ were developed. Their physical properties were then evaluated. The nanocomposite containing PPOH and SBA-15 with a large pore size (SBA-15-L) showed higher toughness, stiffness, and transparency than the other nanocomposites. Our results indicated that the larger pore size of SBA-15-L and the high affinity between PPOH and the MPS surface led to an efficient pore-filled state of SBA-15-L with polymer molecules, forming a nanocomposite with better mechanical strength and transparency.
\end{abstract}

\section{Introduction}

Extensive research and development of polypropylene (PP)based nanocomposites containing nanosized inorganic fillers have been conducted with the aim of improving the physical properties compared with those of composites containing micro-sized fillers [1-10]. In many cases, although stiffness is improved by the dispersion of nanofillers, the presence of nanofillers seriously diminishes toughness owing to a reduction in elongation [10-13]. Lower toughness can be attributed in part to delamination

Electronic supplementary material The online version of this article (https://doi.org/10.1038/s41428-018-0095-x) contains supplementary material, which is available to authorized users.

Ryota Watanabe

r.watanabe@aist.go.jp

$\triangle$ Hideaki Hagihara

h-hagihara@aist.go.jp

1 Research Institute for Sustainable Chemistry, National Institute of Advanced Industrial Science and Technology (AIST), 1-1-1 Higashi, Tsukuba 305-8565, Japan of the filler from the matrix as well as cracking at the grain boundaries of aggregated filler owing to the low adhesion of these materials $[14,15]$. Hence, enhancing the interfacial interactions has great potential to overcome the poor toughness of nanocomposites. For PP nanocomposites, however, improvements to interfacial interactions remain a significant challenge owing to the inertness of PP toward inorganic fillers, which often have a polar nature. As a novel approach to solving this technical problem, we herein propose combining the functionalization of $\mathrm{PP}$ and adoption of mesoporous silica (MPS) filler, which together are likely to create strong interaction points.

Functionalized PPs, such as maleic anhydride-grafted PPs, are commonly used in PP nanocomposites as an interface modifier for improved toughness [16-19]. We have recently developed PP functionalized with hydroxyl groups (PPOH) [20-23] and $\mathrm{PPOH} /$ monodispersed silica nanosphere (SNS) nanocomposite [24-26]. In this case, strong interfacial adhesion between the matrix and filler, which arises from hydrogen bonding, contributes to a marked improvement in tensile strength without a significant loss of elongation. Furthermore, increasing the matrix-filler interfacial area by reducing the SNS particle size improves the reinforcement efficiency of the nanocomposites. 
Fig. 1 FE-SEM images of the fillers. a SBA-15-L, b SBA-15S, c MCM-41, and d SNS; inset shows a high-magnification image
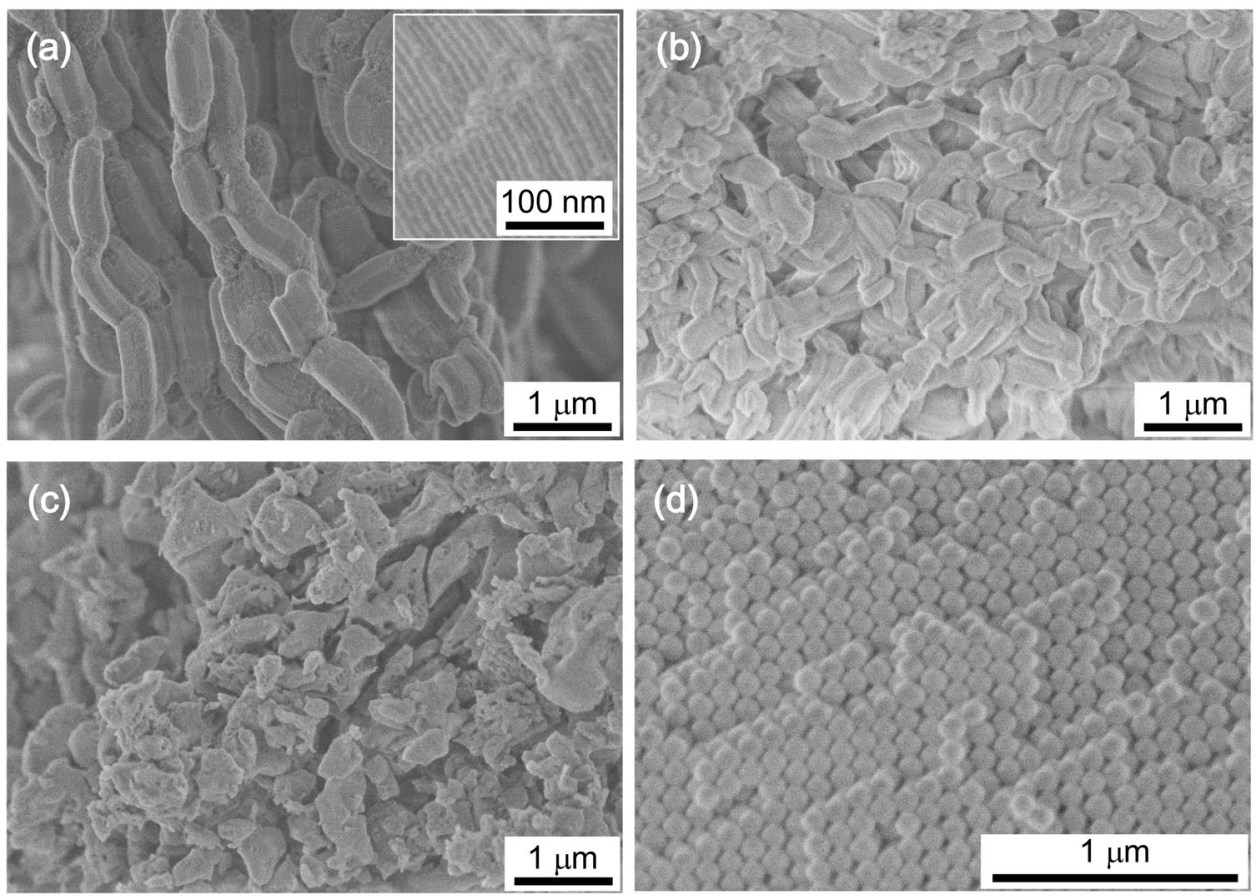

MPS with well-ordered porous structures, and thus an enormously high surface area-to-volume ratio, is expected to be an effective reinforcement filler owing to its strong interfacial interaction derived from the filling of its pores with polymer molecules. PP-based nanocomposites containing MPS have previously been proposed to improve flame retardancy [27, 28]. However, the optimal tradeoff between high toughness and high stiffness that confers outstanding mechanical properties has yet to be attained, because the correlation between the structure and properties of nanocomposites containing MPS remains poorly understood. Characterization of the interaction state between the matrix and MPS, which plays a key role in the physical properties, has not been fully achieved due to difficulties arising from the use of conventional analytical techniques, such as electron microscopy and infrared spectroscopy, to obtain a quantitative analysis of the polymer confined in the small mesopores and of the interfacial interactions. A more revealing investigation of the polymers present in the pores could provide useful information for designing the porosity structure of the material and the required molecular structure of the polymer matrix, leading to improvements in the properties of these nanocomposites.

In this study, we developed nanocomposites composed of MPSs with various porosity structures and PP or $\mathrm{PPOH}$ containing 1.3 or $6.4 \mathrm{~mol} \%$ hydroxyl groups. Three types of MPSs: (i) SBA-15 with large pores $8.0 \mathrm{~nm}$ in size, denoted SBA-15-L; (ii) SBA-15 with small pores $4.4 \mathrm{~nm}$ in size, denoted SBA-15-S; and (iii) MCM-41 as well as nonporous silica nanospheres (SNSs) were used as fillers. The influence of the materials' microstructures on the physical properties of the nanocomposites, such as the morphology of MPS and the chemical structure of the matrix, was evaluated. In the conventional design of composites, increasing the matrix-filler interfacial area by using fillers with a large surface area tends to notably improve the mechanical properties of the materials. In this study, however, nanocomposites containing SBA-15-L exhibited higher mechanical properties and optical transparency than those containing MPS with a large relative surface area. This may be due to more complete filling of the relatively larger pores of SBA-15-L with polymer molecules. In this paper, we describe in detail the influence of the pore structure of MPS and of the hydroxyl group content in the matrix on the physical properties of the nanocomposites. A subsequent paper will address detailed characterization of the matrix-filler interaction and pore-filling structures of the MPS and the nanocomposites.

\section{Experimental}

\section{Materials}

Using previously reported methods [21], PPOH was synthesized with a 5-hexen-1-ol comonomer containing either $1.3 \mathrm{~mol} \%\left(\mathrm{PPOH} 1.3 ; M_{\mathrm{w}}=503,000, M_{\mathrm{w}} / M_{\mathrm{n}}=1.90, \mathrm{~mm}=\right.$ $98.0 \%$ ) or $6.4 \mathrm{~mol} \%$ comonomer (PPOH6.4; $M_{\mathrm{w}}=146,000$, $M_{\mathrm{w}} / M_{\mathrm{n}}=1.95, \mathrm{~mm}=98.4 \%$ ). PP pellets (Novatec ${ }^{\circ} \mathrm{MA} 3$, weight-averaged molecular weight $M_{\mathrm{w}}=397,000$, molecular weight distribution $M_{\mathrm{w}} / M_{\mathrm{n}}=3.57$, isotactic triad $(\mathrm{mm})=93.7 \%)$ were purchased from Japan Polypropylene 
Table 1 Physicochemical properties of SBA-15, MCM-41, and SNS

\begin{tabular}{|c|c|c|c|}
\hline Sample & Particle size $^{\mathrm{a}}$ & Relative surface $\operatorname{area}^{\mathrm{b}}\left(\mathrm{m}^{2} / \mathrm{g}\right)$ & 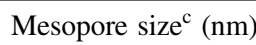 \\
\hline SBA-15-L & $\begin{array}{l}\text { Long axis of over } 2 \mu \mathrm{m} \\
\text { Short axis of } 0.3-0.5 \mu \mathrm{m}\end{array}$ & 619 & 8.0 \\
\hline SBA-15-S & $\begin{array}{l}\text { Long axis of } 0.5-1 \mu \mathrm{m} \\
\text { Short axis of } 0.2-0.5 \mu \mathrm{m}\end{array}$ & 631 & 4.4 \\
\hline MCM-41 & $0.2-1.0 \mu \mathrm{m}$ & 966 & 2.9 \\
\hline SNS & $0.1 \mu \mathrm{m}$ & 100 & - \\
\hline
\end{tabular}

${ }^{a}$ Estimated by SEM images

${ }^{b}$ Estimated using the BET method

${ }^{\mathrm{c}}$ Estimated using the BJH method
Corporation. Mesoporous SBA-15-L, SBA-15-S, and MCM-41 were purchased from Sigma-Aldrich. Uniformly sized SNSs with a mean size of $0.1 \mu \mathrm{m}$ were synthesized using a previously reported method [29].

The morphology of MPS observed by field emission scanning electron microscope (FE-SEM) was shown in Fig. 1a-c. MPS is characterized as micro-sized particles: SBA-15-L (rod-shaped particles with a long axis of over $2 \mu \mathrm{m}$ and a short axis of $0.3-0.5 \mu \mathrm{m})$, SBA-15-S (rod-shaped particles with a long axis of $0.5-1 \mu \mathrm{m}$ and a short axis of $0.2-0.5 \mu \mathrm{m}$ ) and MCM-41 (irregularly shaped particles with a size of $0.2-1.0 \mu \mathrm{m})$. Ordered mesopores were confirmed by FE-SEM imaging (Fig. 1a), and FESEM also confirmed that SNSs consist of highly uniform spheres $0.1 \mu \mathrm{m}$ in size (Fig. 1d).

Table 1 shows the surface area and porosity of the fillers estimated using nitrogen adsorption-desorption isotherms (Figure S1). The surface areas of SBA-15-L and SBA-15-S are almost the same. SBA-15-L and SBA-15-S have different pore sizes of 8.0 and $4.4 \mathrm{~nm}$, respectively. MCM-41 has relatively smaller pores $(2.9 \mathrm{~nm}$ in size) and a larger relative surface area compared with those of SBA15-type MPS. MPS shows a much higher relative surface area than SNSs owing to the presence of porous structures in MPS.

\section{Compounding procedures}

Nanocomposites were prepared by melt-mixing polymer matrices and fillers using a Labo-Plastmill kneading machine (Toyo Seiki Seisakusho) equipped with a KF6 twin rotary mixer $\left(5 \mathrm{~mL}\right.$ in volume) at $180{ }^{\circ} \mathrm{C}$ and $60 \mathrm{rpm}$ for $5 \mathrm{~min}$. The original polymer sample without additional fillers was also melt-mixed to ensure a similar thermal history. The total amount of polymer material, including filler, was $3 \mathrm{~g}$ during each process. No thermal stabilizer was added to the samples. For the structural analyses and mechanical tests, sample sheets $(50 \times 50 \times 0.5 \mathrm{~mm})$ were prepared by hot-pressing the melt-mixed samples at $180^{\circ} \mathrm{C}$ under $5 \mathrm{MPa}$ for $3 \mathrm{~min}$ and then under $15 \mathrm{MPa}$ for $10 \mathrm{~min}$ using a Nafion ${ }^{\circ}$ sheet (Nichias), a stainless window frame (0.5 mm thick) and stainless-steel plates, followed by rapidly quenching to room temperature.

\section{Characterization}

The morphology of the fillers and the filler dispersion in the nanocomposites were observed using an FESEM instrument (S-4800, Hitachi High-Tech Science Corporation) operated at $1 \mathrm{kV}$. The freeze-fractured surfaces of the nanocomposites were observed in back-scattered electron mode without a metal coating. Nitrogen adsorption-desorption measurements were conducted to obtain information about the relative surface area and pore size of the fillers at $-196^{\circ} \mathrm{C}$ using volumetric adsorption measurements (Belsorp mini II, BEL Japan, Inc.). Tensile tests were carried out using a multipurpose stretching tester (EZ-L, Shimadzu) according to ISO 527-1, using dumbbellshaped specimens $(0.5 \mathrm{~mm}$ thick, $4 \mathrm{~mm}$ wide, and $15 \mathrm{~mm}$ long in the parallel region) at a crosshead speed of $10 \mathrm{~mm} /$ min at room temperature. Three specimens were tested for each sample. The elastic modulus was determined from the initial slope in a strain range of $0.1-0.2 \mathrm{~mm}$. The yield strength was taken as the maximum in the yield regime. The area under the curve was used to calculate the tensile toughness. The transmission properties of the sample sheets were measured using a haze meter (NDH5000SP, Nippon Denshoku) in compliance with ISO13468-1.

\section{Results}

\section{Morphological characterization of nanocomposites}

The nanocomposites were fabricated by melt-mixing filler with PP, PPOH1.3, and PPOH6.4. As a representative example of the nanocomposites, the morphologies of the PPOH6.4 nanocomposites were analyzed by FE-SEM. Figure 2 displays SEM images of the areas surrounding the fillers on the cross sections of the sample sheets. A fine dispersion of SBA-15-L in PPOH6.4 is confirmed by the FE-SEM image shown in Fig. 2a. A high-magnification 
(a) PPOH6.4/SBA-15-L

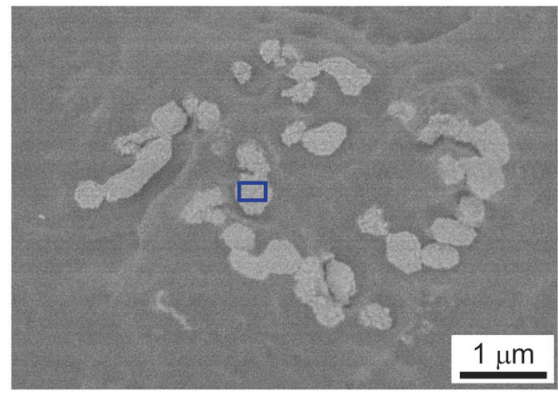

(d) PPOH6.4/MCM-41
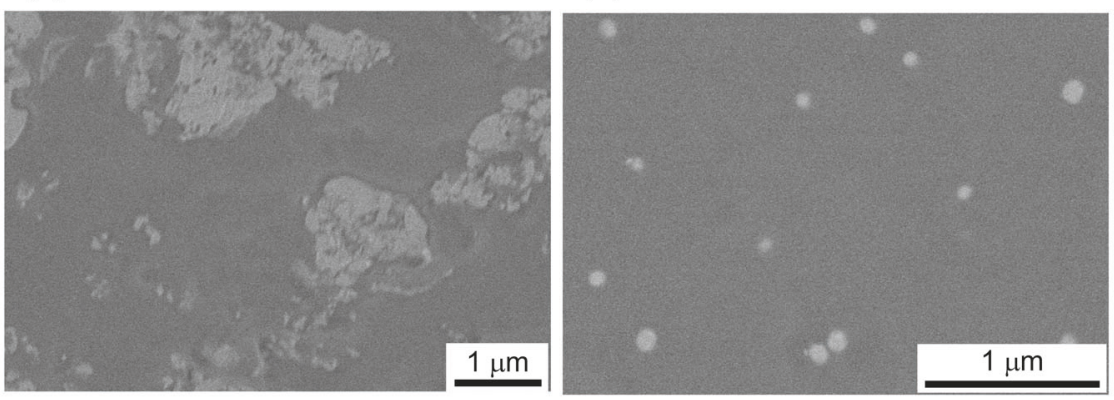

(e) PPOH6.4/SNS

(c) PPOH6.4/SBA-15-S
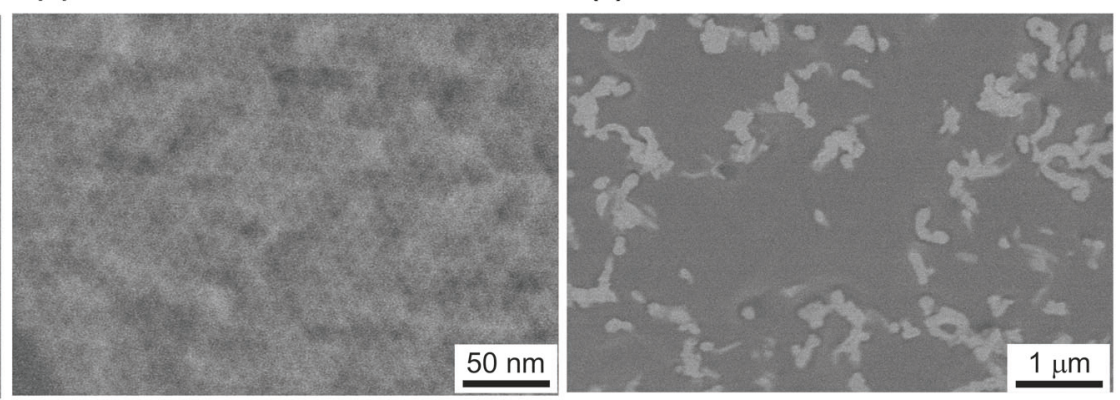

(f) PPOH6.4/SNS
Fig. 2 Cross-sectional FE-SEM images of the nanocomposites; a PPOH6.4/SBA-15-L, b high-magnification image in the area marked by a square in a, c PPOH6.4/SBA-15-S, d PPOH6.4/MCM-41, e PPOH6.4/SNS, and f low-magnification image. The contents of fillers in the present composite are $10 \mathrm{wt} \%$
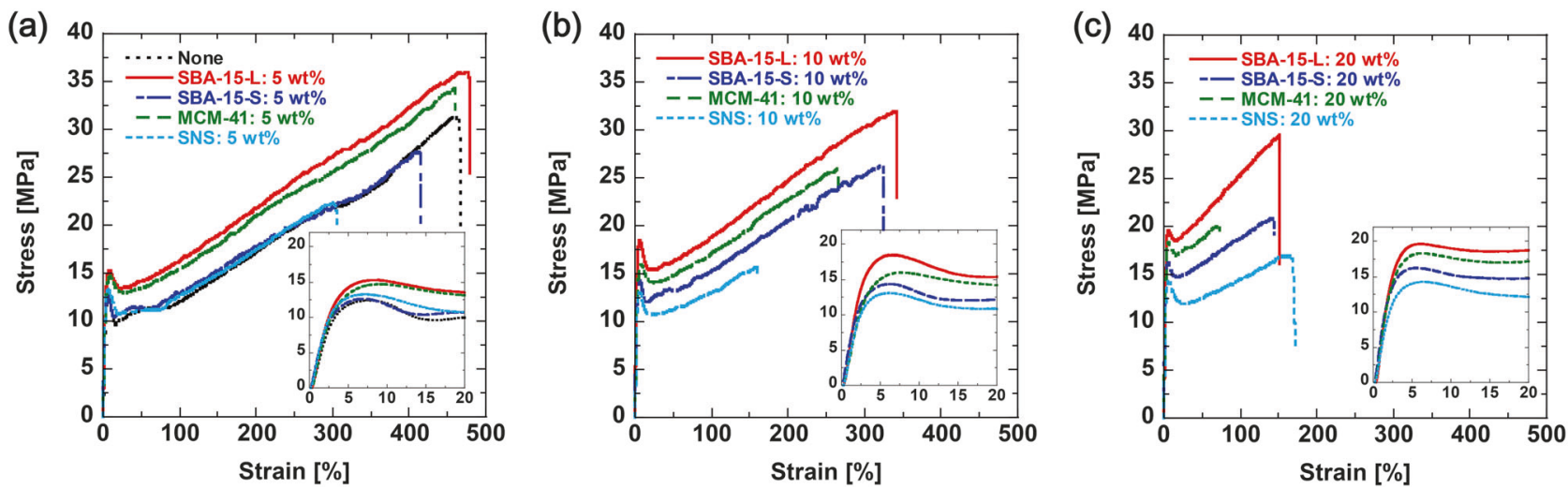

Fig. 3 Stress-strain curves for PPOH6.4 melt-mixed with a 0 and $5 \mathrm{wt} \%$, b $10 \mathrm{wt} \%$, and $\mathbf{c} 20 \mathrm{wt} \%$ filler, such as SBA-15-L, SBA-15-S, MCM-41, and SNSs; insets show magnifications of the initial strain area

FE-SEM image in the area marked by the square in Fig. 2a, focused on SBA-15-L, clearly indicates that the ordered porous structure of SBA-15-L is well preserved, even after composite formation after the melt-mixing process (Fig. 2b). SBA-15-S and MCM-41 were also dispersed in PPOH6.4, as shown in Fig. 2c, d, suggesting the likelihood that MPS functions as a filler with porous structures. A large number of SNSs were uniformly dispersed in a similar manner to that in a previous report (Fig. 2e) [24], whereas slight aggregation of SNSs was observed in the lowmagnification image (Fig. 2f).

\section{Mechanical properties of PPOH6.4 nanocomposites}

First, the effects of the filler structure on the mechanical properties of nanocomposites were examined. Tensile tests were performed on the PPOH6.4 nanocomposites melt-mixed with SBA-15-L, SBA-15-S, MCM-41, and SNSs. The stress-strain curves of PPOH6.4 melt-mixed with $0-20 \mathrm{wt} \%$ filler are shown in Fig. 3. PPOH6.4/SBA15-L exhibits a higher toughness than that of PPOH6.4/ SBA-15-S, PPOH6.4/MCM-41 and PPOH6.4/SNS for all types of filler content. 
Fig. 4 Mechanical properties of PPOH6.4 melt-mixed with 0-20 wt\% filler, such as SBA-15-L, SBA-15-S, MCM-41, and SNSs: a elastic modulus, $\mathbf{b}$ yield strength, c elongation at break, and $\mathbf{d}$ tensile toughness
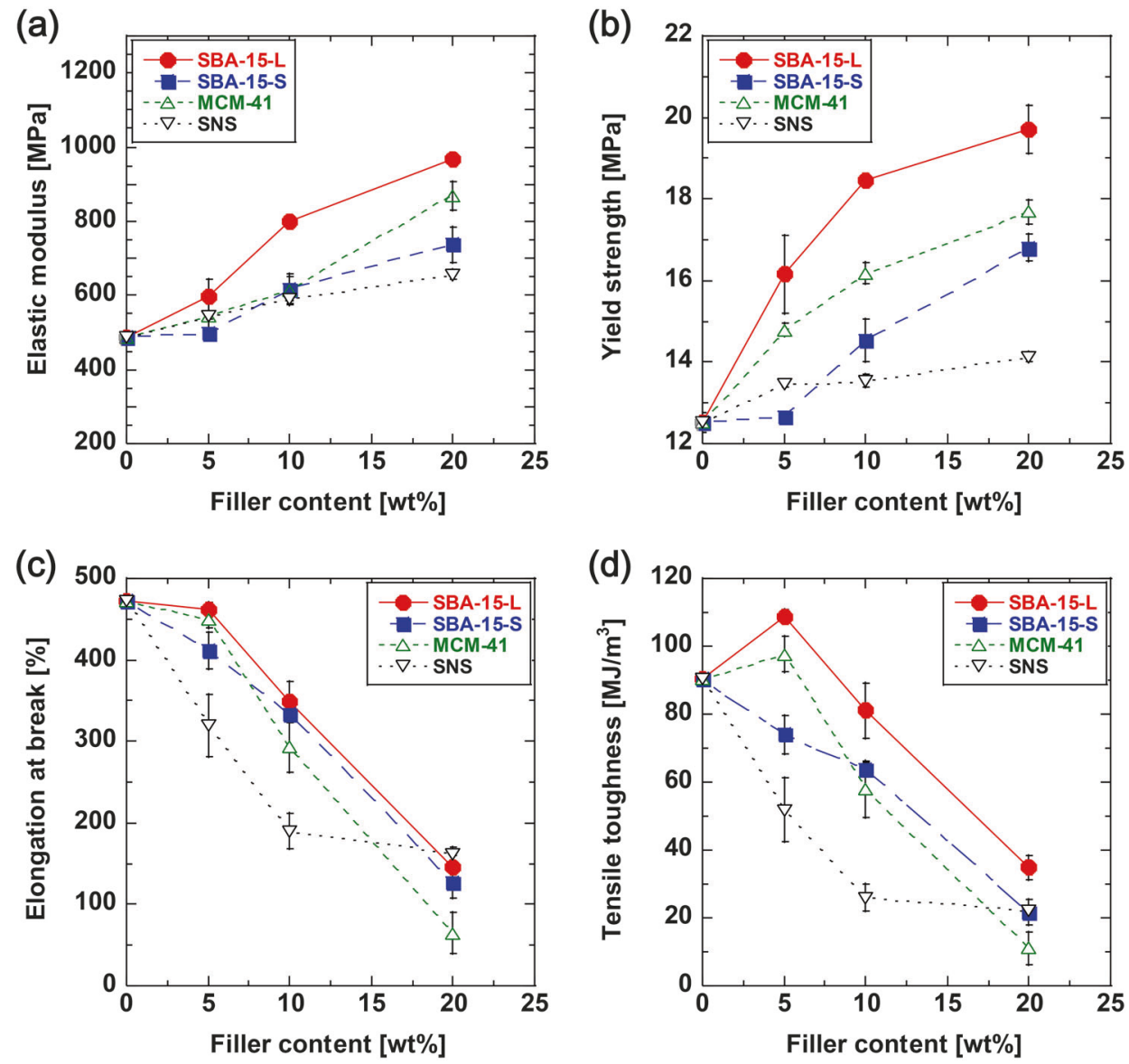

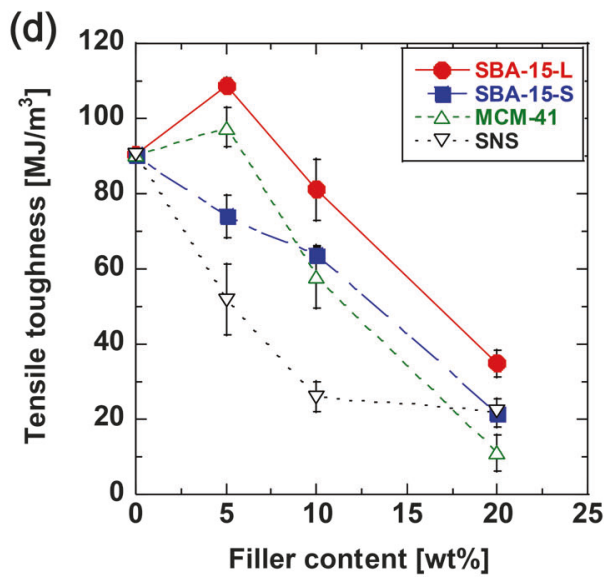

The mechanical properties estimated from the stress-strain curves of the PPOH6.4 nanocomposites are shown in Fig. 4 and Table S1. The elastic modulus and the yield strength increased with the increase in content of filler mixed into PPOH6.4 (Fig. 4a, b). The properties of the nanocomposites containing MPS are basically higher than those containing SNSs, indicating that the presence of a porous structure leads to an increased reinforcement efficiency. The elastic modulus and yield strength of nanocomposites containing MCM-41 are between those of samples containing SBA-15-L and SBA-15-S. Nanocomposites with SBA-15-L in particular provide a higher elastic modulus and a yield strength that exceeds those of nanocomposites with SBA-15-S and MCM-41. As the content of SBA-15-L is increased to $5 \mathrm{wt} \%$, the elongation at break (EB) for the nanocomposite is almost equal to that of the PPOH6.4 matrix, after which the EB continuously decreases as the amount of MPS increases (Fig. 4c). The EB of PPOH6.4/SBA-15-L is slightly higher than that of the other three nanocomposites for each filler content. Figure $4 \mathrm{~d}$ shows the tensile toughness of PPOH6.4 nanocomposites. By melt-mixing with $5 \mathrm{wt} \%$ SBA-15-L, the tensile toughness of PPOH6.4 increases as much as $20 \%$ compared with the toughness of pure PPOH6.4, after which the toughness gradually decreases with further increases in filler content. The tensile toughness of PPOH6.4/SBA-15-L is also greater than that of the other three nanocomposites for each filler content. These values clearly indicate that PPOH6.4/SBA15-L simultaneously provides a better elastic modulus, yield strength, EB and tensile toughness than the other materials.

Next, we investigated the effects of the matrix's chemical structure on the mechanical properties of nanocomposites containing SBA-15-L and SBA-15-S by varying the content of hydroxyl groups in the matrix. Figure 5 and Table S1 show the mechanical properties of the nanocomposites of PP and PPOH1.3 melt-mixed with 0-20 wt\% SBA-15-L and SBA-15-S. The elastic moduli of the PP and PPOH1.3 nanocomposites increased continuously with increasing MPS content in a similar manner to that seen for the PPOH6.4 nanocomposite (Fig. 5a, c). The elastic modulus of SBA-15-L is higher than that of SBA-15-S at each filler content. The EB of the PPOH1.3 nanocomposites continuously decreases with increasing filler content (Fig. 5b). The EB for PPOH1.3/SBA-15-L is almost equal to that of the PPOH1.3 matrix and higher than that for PPOH1.3/ SBA-15-S with $5 \mathrm{wt} \%$ filler, after which the values steeply decrease with any further increase in filler content. Here, the EB of PPOH1.3 with over $10 \mathrm{wt} \%$ filler is much smaller 
Fig. 5 Mechanical properties of PPOH1.3 (top) and PP (bottom) melt-mixed with SBA-15-L and SBA-15-S. a, c Elastic modulus and $\mathbf{b}, \mathbf{d}$ elongation at break
Fig. 6 Effects of the hydroxyl content in a polymer on the tensile toughness of the original polymers and nanocomposites containing SBA-15-L and SBA15-S. Nanocomposites were melt-mixed with a $5 \mathrm{wt} \%$ and b $10 \mathrm{wt} \%$ filler
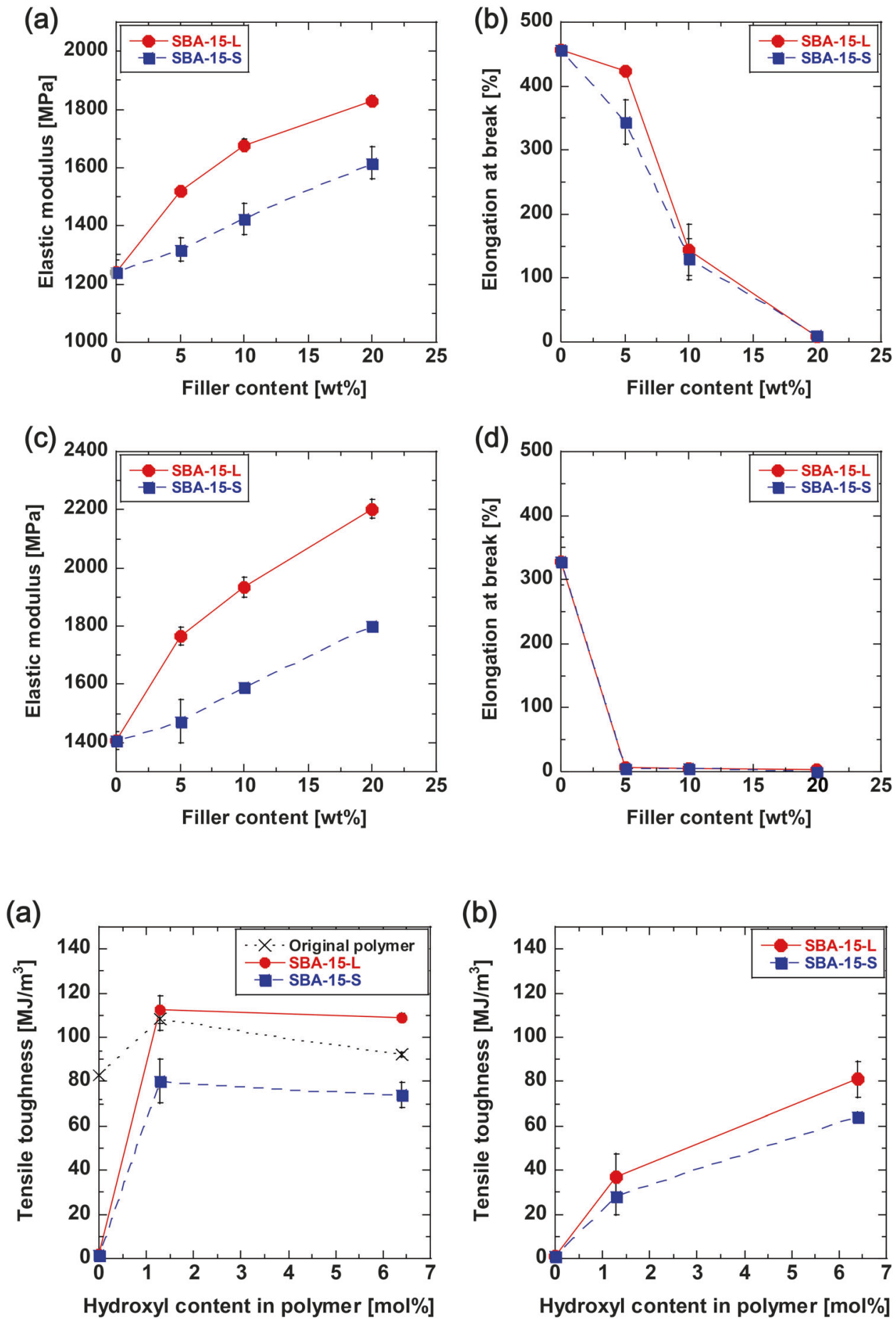

(b)

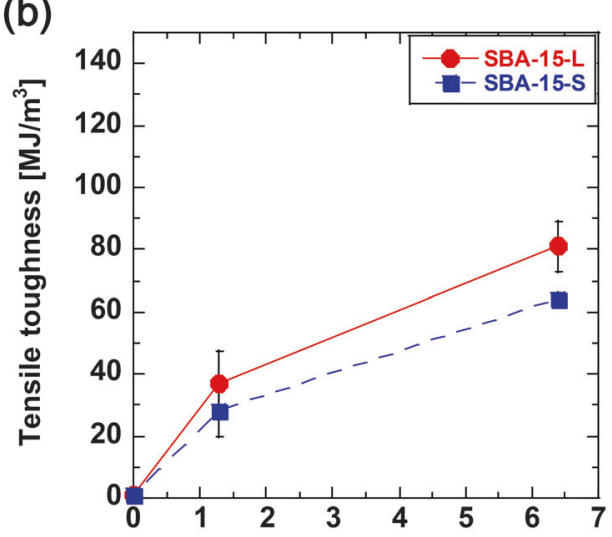

Hydroxyl content in polymer [mol\%] than that for PPOH6.4 with over $10 \mathrm{wt} \%$ fillers. The EB for PP (329\%) significantly falls to below $10 \%$ with only a $5 \mathrm{wt} \%$ filler addition, suggesting a lack of any interfacial adhesion between inert PP and the fillers (Fig. 5d). The crystallinities and melting points of polymer matrices in SBA-15-L nanocomposites, which can affect the mechanical properties, are shown in Figure S3. The crystallinity of PPOH6.4 decreased as the content of SBA-15-L increased from 20.4 to $17.0 \%$, whereas the crystallinity of PPOH6.4 was almost unchanged by the addition of MCM-41 and SNSs. The addition of SBA-15-L did not lead to a change in the melting point.

The influence of the $\mathrm{OH}$-comonomer content in the matrix on the tensile toughness of the original polymers and the nanocomposites melt-mixed with 5 and $10 \mathrm{wt} \%$ filler is shown in Fig. 6. The tensile toughness values of the original PP, PPOH1.3, and PPOH6.4 are very similar. The tensile toughness of non-functionalized PP is almost always 

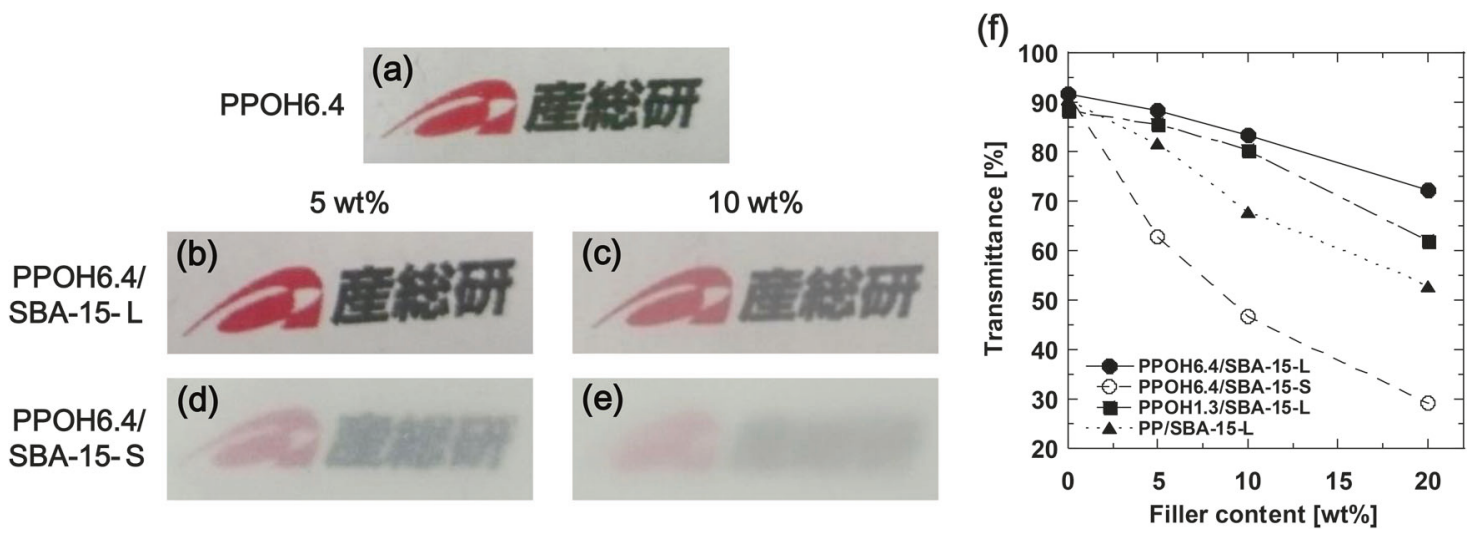

Fig. 7 Photographs of sample sheets $0.5 \mathrm{~mm}$ thick of a PPOH6.4 matrix, b and $\mathbf{c}$ PPOH6.4/SBA-15-L, and d and e PPOH6.4/SBA-15$\mathrm{S}$. The contents of fillers are $5 \mathrm{wt} \%$ (left) and $10 \mathrm{wt} \%$ (right). The

weakened by the addition of fillers owing to a lack of ductility, whereas the tensile toughness of nanocomposites containing SBA-15-L and SBA-15-S increases with additional hydroxyl groups in the PP polymer chain. The difference in tensile toughness between SBA-15-L and SBA-15-S widens as the hydroxyl content is increased.

\section{Optical properties of the nanocomposites}

Figure 6a-e are photographic images of sample sheets (thickness: $0.5 \mathrm{~mm}$ ) of PPOH6.4 and its nanocomposites containing 5 and $10 \mathrm{wt} \%$ fillers. The transparency of PPOH6.4 is almost fully maintained after melt-mixing 5 and $10 \mathrm{wt} \%$ SBA-15-L, whereas the introduction of SBA-15-S significantly decreases transparency. Photographs of sample sheets of PPOH6.4/SNS are provided in Figure S4. The addition of SNSs decreases the transparency of the sample sheets presumably owing to the existence of SNS aggregation and the difference in the refractive index of polymer matrix and that of silica. We also evaluated the transparency of PP and the PPOH1.3 and PPOH6.4 nanocomposites using a haze meter (Fig. 7). PPOH6.4/SBA-15-L maintained a high transmittance of $72 \%$ even after the addition of $20 \mathrm{wt} \%$ filler, whereas the transmittance of PPOH6.4/ SBA-15-S fell steeply to $29 \%$ as the filler content increased to $20 \mathrm{wt} \%$. The transmittance of PP/SBA-15-L showed a slight decrease, although it is higher than that of PPOH6.4/ SBA-15-S.

\section{Discussion}

Tensile tests reveal that the reinforcement performance of MPS depends on the pore structure [30]. PPOH6.4/SBA-15L shows an effectively improved elastic modulus and minimizes the reduction in ductility and toughness of the original polymer. In the conventional design process, sample sheets were placed directly on the background. f Total transmittance of the nanocomposites containing SBA-15-L and SBA-15-S

enlarging the matrix-filler interfacial area using fillers with a large surface area is expected to effectively improve the mechanical properties. In contrast, the PPOH6.4/SBA-15-L exhibits higher toughness and stiffness than PPOH6.4/ MCM-41 despite the relative surface area of SBA-15-L $\left(619 \mathrm{~m}^{2} / \mathrm{g}\right)$ being smaller than that of MCM-41 $\left(966 \mathrm{~m}^{2} / \mathrm{g}\right)$. This result implies that the specific properties of PPOH6.4/ SBA-15-L are partly attributable to the formation of the filled-pore state. The relatively large pore size of SBA-15-L likely contributes to the high efficiency of the insertion of polymer molecules into the pores, leading to a strong interfacial interaction. Regardless of the content of hydroxyl groups in the matrix, the tensile toughness and stiffness of nanocomposites containing SBA-15-L are higher than those of nanocomposites containing SBA-15-S. Moreover, the tensile toughness of nanocomposites with $10 \mathrm{wt} \%$ SBA-15$\mathrm{L}$ increased as the hydroxyl content of the matrix increased, possibly indicating that the efficiency of pore-filling of SBA-15-L is also dependent on the hydroxyl group content. In the case of the difference in the elastic modulus and yield strength between the nanocomposites containing SBA-15-S and MCM-41, structural factors in addition to the pore size influence the mechanical properties of the nanocomposites. For example, the difference in the porous structure between SBA-15-S and MCM-41 could affect the mechanical properties.

The pore-filling of MPS in the matrix is likely related to the optical transparency of the nanocomposites. A loss in transparency is owing to scattering by filler particles in the polymer matrix and refractive index mismatch. The loss of transparency due to Rayleigh scattering can be described by equation (1) [31]

$$
T_{\mathrm{PNC}}=0.9 \times \exp \left\{-\frac{32 \pi^{4} \Phi_{p} x r^{3} n_{m}^{4}}{\lambda^{4}}\left[\frac{\left(\frac{n_{p}}{n_{m}}\right)^{2}-1}{\left(\frac{n_{p}}{n_{m}}\right)^{2}+2}\right]\right\}
$$


where $T_{\mathrm{PNC}}$ is the transmittance of polymer nanocomposites, $\lambda$ is the wavelength of incident light, $x$ is the optical path length, $\Phi_{\mathrm{p}}$ is the volume fraction of the particles, $r$ is the size of particles, $n_{\mathrm{p}}$ and $n_{\mathrm{m}}$ are the refractive index of the particles and the matrix, respectively. The transparency loss increases exponentially with increasing volume fraction and with particle size. In addition, if the refractive indices $n_{\mathrm{p}}$ and $n_{\mathrm{m}}$ are the same, the scattering intensity is zero and is independent of the particle size. MPS particles basically show a very low refractive index $\left(n_{\mathrm{p}}=1.09-1.16\right)$ [32], resulting in the refractive index mismatch between MPS and PP matrices $\left(n_{\mathrm{m}}=1.49\right)$. Even with the small refractive index mismatch (0.12) of nanocomposite composed of $\mathrm{CeF}_{3}$ particles and polymethyl methacrylate, the transparency decreased rapidly as the particle loading was increased to $10 \mathrm{vol} \%$ [33]. Therefore, the MPS particles with unfilled pores caused light scattering that resulted in a loss of transparency. Despite the relatively large mismatch of the refractive index between MPS and PP ( 0.4), the PPOH6.4 composites maintain a high transmittance after melt-mixing with SBA-15-L. This implies that $r$ is decreased and approaches the pore-wall thickness of SBA-15-L by the effective pore-filling of SBA-15-L. In addition, the loss of transmittance by melt-mixing with SBA-15-S suggests insufficient pore-filling of SBA-15-S with the matrix. PPOH1.3/SBA-15-L also maintains a high transmittance in a similar manner to that of the PPOH6.4 nanocomposite. The slight decrease in transmittance of PP/ SBA-15-L suggests that a proportion of the pores remain unfilled due to low affinity between PP and SBA-15-L.

By combining the results from the measurements of mechanical and optical properties, it is possible to suggest that the relatively large pores of SBA-15-L are efficiently filled with the matrix, whereas the smaller pores of SBA-15-S are less efficiently filled. In general, insertion of polymer chains into the mesopores is assumed to be affected by both the dimension of the pore and the wettability of the pore surface. The introduction of hydroxyl groups into PP likely induces a surface energy closer to that of MPS, resulting in improved wettability. Hydrogen bonding between PPOH and MPS is also expected to be helpful for the stabilization of the pore-filling state. Furthermore, the large pore diameter of SBA-15-L allows the $\mathrm{PPOH}$ chains to be easily inserted into the pores, whereas the insertion of the polymer into the small pores of other MPSs are limited owing to a loss of entropy. The pore-filling efficiency and interfacial interactions between polymers and pores of MPS appear to contribute to anchoring the polymer molecules to the fillers, leading to improved stiffness and toughness.

\section{Conclusions}

Nanocomposites were fabricated using MPS with various pore sizes (two types of SBA-15 with pore sizes of 4.4 and
$8.0 \mathrm{~nm}$, and MCM-41 with a pore size of $2.9 \mathrm{~nm}$ ) or nonporous SNSs, which were melt-mixed into $\mathrm{PP}$ or $\mathrm{PPOH}$ with varying hydroxyl group contents (1.3 or $6.4 \mathrm{~mol} \%$ ). The PPOH/SBA-15-L simultaneously provided higher tensile toughness, elastic modulus, and transparency than seen in the other nanocomposites. It was also revealed that the tensile toughness of the nanocomposites containing SBA15-L increased with the increase in hydroxyl group content of the matrix. The transparency of PPOH was almost fully maintained after melt-mixing SBA-15-L, whereas it was significantly decreased by melt-mixing SBA-15-S and MCM-41. Our results suggest that sufficient pore-filling of MPS with the matrix leads to improved mechanical and optical properties. A close examination of the pore-filling state in nanocomposites is key to improving the properties of nanocomposites containing MPS. Our second paper describes newly developed evaluation techniques for both matrix-filler interactions and the pore-filling state of MPS using temperature-programmed pyrolysis.

Acknowledgements This work was financially supported by the Izumi Science and Technology Foundation.

\section{Compliance with ethical standards}

Conflict of interest The author declares that he has no conflict of interest.

Open Access This article is licensed under a Creative Commons Attribution 4.0 International License, which permits use, sharing, adaptation, distribution and reproduction in any medium or format, as long as you give appropriate credit to the original author(s) and the source, provide a link to the Creative Commons license, and indicate if changes were made. The images or other third party material in this article are included in the article's Creative Commons license, unless indicated otherwise in a credit line to the material. If material is not included in the article's Creative Commons license and your intended use is not permitted by statutory regulation or exceeds the permitted use, you will need to obtain permission directly from the copyright holder. To view a copy of this license, visit http://creativecommons. org/licenses/by/4.0/.

\section{References}

1. Ray SS, Okamoto M. Polymer/layered silicate nanocomposites: a review from preparation to processing. Prog Polym Sci. 2003;28: 1539-641.

2. Okada A, Usuki A. Twenty years of polymer-clay nanocomposites. Macromol Mater Eng. 2006;291:1449-76.

3. Chan CM, Wu JS, Li JX, Cheung YK. Polypropylene/calcium carbonate nanocomposites. Polymer. 2002;43:2981-92.

4. Hasegawa N, Okamoto H, Kato M, Usuki A. Preparation and mechanical properties of polypropylene-clay hybrids based on modified polyproplene and organophilic clay. J Appl Polym Sci. 2000;78:1918-22.

5. Fu SY, Feng XQ, Lauke B, Mai YW. Effects of particle size, particle/matrix interface adhesion and particle loading on mechanical properties of particulate-polymer composites. Compos Part B Eng. 2008;39:933-61. 
6. Iwamoto S, Yamamoto S, Lee SH, Endo T. Mechanical properties of polypropylene composites reinforced by surface-coated microfibrillated cellurlose. Compos Part A Appl Sci Manuf. 2014;59:26-29.

7. Dougnac VN, Alamillo R, Peoples BC, Quijada R. Effect of particle diameter on the permeability of polypropylene/SiO nanocomposites. Polymer. 2010;51:2918-26.

8. Rong MZ, Zhang MQ, Zheng YX, Zeng HM, Friedrich $\mathrm{K}$. Improvement of tensile properties of nano- $\mathrm{SiO}_{2} / \mathrm{PP}$ composites in relation to percolation. Polymer. 2001;42:3301-4.

9. Song P, Cao Z, Cai Y, Zhao L, Fang Z, Fu S. Fabrication of exfoliated graphene-based polypropylene nanocomposites with enhanced mechanical and thermal properties. Polymer. 2011;52: 4001-10.

10. Taniike T, Toyonaga M, Terano M. Polypropylene-grafted nanoparticles as a promising strategy for boosting physical properties of polypropylene-based nanocomposites. Polymer. 2014;55:1012-9.

11. Bikiaris DN, Papageorgiou GZ, Pavlidou E, Vouroutzis N, Palatzoglou P, Karayannidis GP. Preparation by melt mixing and characterization of isotactic polypropylene/ $\mathrm{SiO}_{2}$ nanocomposites containing untreated and surface-treated nanoparticles. J Appl Polym Sci. 2006;100:2684-96.

12. Iyer KA, Torkelson JM. Importance of superior dispersion versus filler surface modification in producing robust polymer nanocomposites: the example of polypropylene/nanosilica hybrids. Polymer. 2015;68:147-57.

13. Bikiaris D, Vassiliou A, Chrissafis K, Paraskevopoulos KM, Jannakoudakis A, Docoslis A. Effect of acid treated multi-walled carbon nanotubes on the mechanical, permeability, thermal properties and thermo-oxidative stability of isotactic polypropylene. Polym Degrad Stab. 2008;93:952-67.

14. Zuiderduin WCJ, Westzaan C, Huetink J, Gaymans RJ. Toughening of polypropylene with calcium carbonate particles. Polymer. 2003;44:261-75.

15. Tjong SC. Structural and mechanical properties of polymer nanocomposites. Mater Sci Eng R. 2006;53:73-197.

16. Bikiaris DN, Vassiliou A, Pavlidou E, Karayannidis GP. Compatibilisation effect of PP-g-MA copolymer on iPP/SiO ${ }_{2}$ nanocomposites prepared by melt mixing. Eur Polym J. 2005;41: 1965-78.

17. Svoboda P, Zeng C, Wang H, Lee LJ, Tomasko DL. Morphology and mechanical properties of polypropylene/organoclay nanocomposites. J Appl Polym Sci. 2002;85:1562-70.

18. Ton-That MT, Perrin-Sarazin F, Cole KC, Bureau MN, Denault J. Polyolefin nanocomposites: formulation and development. Polym Eng Sci. 2004;44:1212-9.

19. Chen L, Wong SC, Pisharath S. Fracture properties of nanoclay-filled polypropylene. J Appl Polym Sci. 2003;88: 3298-305.

20. Hagihara H, Ishihara T, Hoang TB, Shiono T. Precise control of microstructure of functionalized polypropylene synthesized by the ansa-zirconocene/MAOcatalysts. J Polym Sci Part A Polym Chem. 2008;46:1738-48.
21. Iizuka Y, Sugiyama J, Hagihara H. Unexpected mechanical properties of functionalized polypropylene: tensile test, Charpy impact tensile test, DSC, and WAXD analysis of poly(5-hexen-1ol-co-propylene). Macromolecules. 2009;42:2321-3.

22. Hagihara H, Ito K, Kimata S. Comprehensive study of altered amorphous structure in functionalized polypropylenes exhibiting high tensile strength. Macromolecules. 2013;46:4432-7.

23. Hagihara H, Tsuchihara K, Takeuchi K, Murata M, Ozaki H, Shiono T. Copolymerization of ethylene or propylene with $\alpha$ olefins containing hydroxyl groups with zirconocene/methylaluminoxane catalyst. J Polym Sci Part A Polym Chem. 2004;42: $52-8$.

24. Watanabe R, Kunioka M, Mizukado J, Suda H, Hagihara H. Highly ductile polypropylene-based nanocomposites by dispersing monodisperse silica nanospheres in functionalized polypropylene containing hydroxyl groups. Polymer. 2016;99:63-71.

25. Watanabe R, Shinzawa H, Kunioka M, Mizukado J, Suda H, Hagihara H. Reinforcement mechanism of functionalized polypropylene containing hydroxyl group nanocomposites studied by rheo-optical near-infrared spectroscopy. Eur Polym J. 2017;92: 86-96.

26. Watanabe R, Kunioka M, Sato H, Mizukado J, Hagihara H. Management of both toughness and stiffness of polypropylene nanocomposites using poly(5-hexen-1-ol-co-propylene) and silica nanospheres. Polym Adv Technol. 2018;1:417-23.

27. Wang N, Zhang J, Fang Q, Hui D. Influence of mesoporous fillers with PP- $g$-MA on flammability and tensile behavior of polypropylen composites. Compos Part B Eng. 2013;44:467-71.

28. Wang N, Gao N, Jiang S, Fang Q, Chen E. Effect of different structure MCM-41 fillers with PP-g-MA on mechanical and crystallization performances of polypropylene. Compos Part B Eng. 2011;42:1571-7.

29. Watanabe R, Yokoi T, Kobayashi E, Otsuka Y, Shimojima A, Okubo T, Tatsumi T. Extension of size of monodisperse silica nanospheres and their well-ordered assembly. J Colloid Interface Sci. 2011;360:1-7.

30. The FE-SEM observation of the fillers indicates the particle size of MPS is varied in the micrometer and sub-micrometer range. In general, such difference in the particle size does not exert enormous influence on mechanical properties of polymer composites [5]. The ordered porosity of SBA-15-L was preserved even after melt-mixing with PPOH6.4. These suggests that the performance of MPS as filler is mainly influenced by the difference in the porous structure of MPS.

31. Tao P, Viswanath A, Li Y, Siegel RW, Benicewicz BB, Schadler LS. Bulk transparent epoxy nanocomposites filled with poly (glycidyl methacrylate) brush-grafted $\mathrm{TiO}_{2}$ nanoparticles. Polymer. 2013;54:1639-46.

32. Yamaguchi M, Nakayama H, Yamada K, Imai H. Ultralo refractive index coatings consisting of mesoporous silica nanoparteicles. Opt Lett. 2009;34:2060-2.

33. Tan MC, Patil SD, Riman RE. Transparent infrared-emitting $\mathrm{CeF}_{3}$ : $\mathrm{Yb}$-Er polymer nanocomposites for optical applications. ACS Appl Mater Interfaces. 2010;7:1884-91. 